\title{
A Quadrupole/Time-of-Flight Mass Spectrometry Study of Trp-Cage's Conformation
}

\author{
Mingxiang Lin, Zeeshan Ahmed, Christopher R. Taormina, \\ and Kasi V. Somayajula* \\ Department of Chemistry, University of Pittsburgh, Pittsburgh, Pennsylvania, USA
}

\begin{abstract}
Trp-cage is a synthetic 20-residue miniprotein that uses tertiary contacts to stabilize its native conformation. NMR, circular dichroism (CD), and UV-resonance Raman spectroscopy were used to probe its energy landscape. In this quadrupole/time-of-flight study, electrospray ionization charge state distribution (CSD) and solution-phase H/D exchange are used to probe Trp-cage's tertiary structure. The CSDs of Trp-cage and its mutant provide spectra showing a $\mathrm{pH}$-dependent conformation change. Solution-phase $\mathrm{H} / \mathrm{D}$ exchange in $30 \%$ deuterated trifluoroethanol solution of the wild type shows increased protection of one labile hydrogen in the native state. Together, CSDs and solution-phase H/D exchange are demonstrated to constitute a simple but effective means to follow conformation changes in a small tertiary protein. (J Am Soc Mass Spectrom 2007, 18, 195-200) (C) 2007 American Society for Mass Spectrometry
\end{abstract}

$\mathrm{T}$ Trp-cage is a synthetic 20-residue miniprotein of sequence $\mathrm{N}_{1}$ LYIQWLKDG ${ }_{10}$ GPSSGRPPPS, which resulted from a de novo design effort [1]. The effort began with an examination of a poorly folded 39 residue long saliva protein of a Gila monster. Using an iterative design effort with selective mutations and truncations, Neidigh et al. [1] created different variants and characterized their folded states using NMR and circular dichroism spectroscopy (CD). Trp-cage, originally referred to as $\mathrm{Tc} 5 \mathrm{~b}$, exhibited the most structure in terms of the cage motif. It was found to be $95 \%$ folded under physiological conditions.

Trp-cage consists entirely of natural amino acids and has been shown to exhibit elements of tertiary structure in the absence of disulfide bridges, metal ion chelation, or stabilization through oligomerization [2]. NMR and CD data of Neidigh et al. [1] suggest a simple two-state unfolding mechanism. Using the intrinsic fluorescence of Trp, Qiu et al. [3] determined the folding time to be $4 \mu \mathrm{s}$, which makes Trp-cage the fastest folding polypeptide to exhibit a tertiary structure.

Several groups previously reported theoretical simulations of Trp-cage's folding [4-11]. Simmerling et al. [4], in their all atom molecular dynamics simulation, calculated a native-state topology consistent with the

Published online October 24, 2006

Address reprint requests to C. Taormina, Department of Chemistry, University of Pittsburgh, Pittsburgh, PA, 15260, USA. E-mail: crtst7@pitt.edu

* Present address: The Coca-Cola Company, 1 Coca-Cola Plaza, Atlanta, GA 30313
NMR structure reported by Neidigh et al. [1]. Their simulation at $325 \mathrm{~K}$, which modeled the folding of Trp-cage, found that it converges to a native-state topology within 20 ns. Snow et al. [6] carried out stochastic dynamics simulations over a total modeled folding time of $100 \mu$ s and found that the unfolded state retains features resembling the native-state topology.

Ahmed et al. [12] recently $^{\circ}$ used $^{\circ} \mathrm{UV}$-resonance ${ }^{\circ} \mathrm{Ra}-$ man spectroscopy (UVRR) to examine the thermal unfolding behavior of Trp-cage. The authors demonstrated that at physiological $\mathrm{pH}$, Trp-cage initially melts to a more compact intermediate state as temperature is increased from 4 to $20^{\circ} \mathrm{C}$, while retaining its nativestate secondary structure. Increasing the temperature to $70{ }^{\circ} \mathrm{C}$ results in partial unfolding of this maximally compact intermediate, although it still retains some native-like features even at high temperatures, as suggested by the invariance of the trytophan's $\chi^{2}$ dihedral angle $[12]$.

Recently, ${ }^{\circ} \mathrm{Adams}^{\circ} \mathrm{et}^{\circ} \mathrm{al}^{\circ} .^{\circ}[13]^{\circ}$ applied $^{\circ}$ electron ${ }^{\circ}$ capture dissociation mass spectrometry to examine the conformation of different charge states of Trp-cage. The authors propose that the +2 state retains the solutionphase native conformation, whereas the +3 charge state, dominated by ionic hydrogen bonds, adopts a more open gas-phase conformation. Furthermore, Adams $^{\circ}$ et $^{\circ}$ al. $^{\circ}[13]^{\circ}$ demonstrated $^{\circ}$ that $^{\circ}$ D-Tyr ${ }^{\circ}$ substitution denatures Trp-cage's native structure. The D-isomer prevents stabilization of the tryptophan side chain that destabilizes the hydrogen-bond network and increases the ${ }^{\circ}$ interior $^{\circ}$ solvent ${ }^{\circ}$ accessibility $^{\circ}[13]$. 
In this study, we use the conformational dependency of protein charge state distributions (CSDs) in electrospray ionization mass spectrometry (ESI-MS) to examine the $\mathrm{pH}$ dependency of Trp-cage's conformation. Well documented for over a decade, protein CSDs in ESI-MS show a shift toward higher charge state values upon

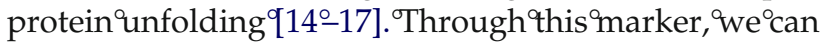
discriminate between different global conformations of Trp-cage as the solvent $\mathrm{pH}$ is varied. Furthermore, we also probe Trp-cage conformation by solution-phase hydrogen/deuterium exchange, which is an established mass spectrometric technique for protein conformation and $^{\circ}$ dynamics ${ }^{\circ}$ studies $^{\circ}[18]$.

\section{Experimental}

Mass spectrometry measurements were performed on a hybrid quadrupole time-of-flight mass spectrometer (Q-ToF MS, API-US, Micromass UK Ltd., Manchester, UK), equipped with a Z-spray electrospray source. The typical instrument operation parameters at the source region for this study were: capillary voltage $3.5 \mathrm{kV}$, cone voltage $40 \mathrm{~V}$, source temperature $80^{\circ} \mathrm{C}$, desolvation temperature $150{ }^{\circ} \mathrm{C}$, cone gas flow $60 \mathrm{~L} / \mathrm{h}$, and desolvation gas flow $600 \mathrm{~L} / \mathrm{h}$. Sample solutions were injected from the embedded syringe pump at a flow rate of $10 \mu \mathrm{L} / \mathrm{min}$. Argon was used as the collision gas with a pressure of $5 \times 10^{-5}$ bar at the analyzer pressure. The collision energy was kept at $10 \mathrm{~V}$ in the ToF MS mode. The instrument was calibrated externally by a cesium iodide solution up to $2500 \mathrm{amu}$. Spectra were accumulated at $1 \mathrm{~s}$ per scan at reflectron $\mathrm{V}$ mode with a full-width half-maximum resolution of 10,000. Data acquisition and processing were performed by use of MassLynx V4.0 software (Waters Corp., Milford, MA).

Trp-cage peptide was obtained at $>95 \%$ purity from the Pittsburgh peptide synthesis facility and was used at $40 \mu \mathrm{M}$ concentrations. D-Tyr substituted Trp-cage peptide was provided by Dr. Zubarev at Uppsala University and was used at $18 \mu \mathrm{M}$ concentrations. The $\mathrm{pH}$ in all of the experiments was adjusted using glacial acetic acid (Aldrich, St. Louis, MO) and $\mathrm{NH}_{4} \mathrm{OH}$ (Aldrich).

Charge state distribution analysis was carried out by measuring the intensity differences of the ToF spectrum of Trp-cage aqueous solutions at $\mathrm{pH} 3$ and $\mathrm{pH}$ 7. A complete deuterium-exchanged Trp-cage spectrum was obtained from a $100 \% \mathrm{D}_{2} \mathrm{O}$ Trp-cage solution buffered at $\mathrm{pH} 7$ using deuterated acetic acid and ammonium hydroxide (Sigma Chemical, St. Louis, MO). By dissolving Trp-cage directly in $70 \% \mathrm{D}_{2} \mathrm{O}$ and $30 \%$ deuterated trifluoroethanol, $\mathrm{CF}_{3} \mathrm{CH}_{2} \mathrm{OD}$ (d-TFE, Sigma Chemical), we examined the amide hydrogen protection against hydrogen-deuterium (H/D) exchange in a 30\% TFE (Aldrich) solution by continuously monitoring the temporal dependency of the spectrum up to $30 \mathrm{~min}$.

\section{Results and Discussion}

Trp-cage charge state distributions were obtained at $\mathrm{pH}$ $3^{\circ}$ and $^{\circ} \mathrm{pH}^{\circ}$ 7. ${ }^{\circ} \mathrm{As}^{\circ}$ shown $^{\circ}$ in $^{\circ}$ Figure $^{\circ} 1 \mathrm{a}^{\circ}{ }^{\circ}$ the ${ }^{\circ} \mathrm{ESI}^{\circ}$ mass spectrum of the wild-type Trp-cage at $\mathrm{pH} 7$ shows three peaks corresponding to the $+1,+2$, and +3 charge state, with +2 being the dominant state. The intensity ratio of charge state +2 /charge state +3 is 2.0. The intensity ratios obtained at different solvent conditions and $^{\circ} \mathrm{pH}^{\circ}$ values $^{\circ}$ are $^{\circ}$ summarized $^{\circ}$ in $^{\circ}$ Table $^{\circ} 1 .^{\circ}$ As $^{\circ}$ the solution $\mathrm{pH}$ is decreased from $\mathrm{pH} 7$ to $\mathrm{pH} 3$, the CSD shifts from the +2 to the +3 state and the intensity ratio of +2 to +3 charge states decreases from 2.0 to 0.3 . The weak +1 state observed at $\mathrm{pH} 7$ is no longer observed at $\mathrm{pH}$ 3. Our results indicate that at low $\mathrm{pH}$ Trp-cage denatures, adopting a more open conformation results in a CSD shift to the +3 charge state. Contrary to the behavior of wild-type Trp-cage, the D-Tyr substituted mutant shows a dominant +3 state at either $\mathrm{pH}$ with the intensity ratio of +2 to +3 charge states being 0.2 at $\mathrm{pH} 3$ and 0.6 at $\mathrm{pH}$ 7. The spectrum obtained at $\mathrm{pH} 7$ is shown ${ }^{\circ}{ }^{\circ}{ }^{\circ}$ Figure $^{\circ} 2 b .{ }^{\circ}$ The $^{\circ}$ difference ${ }^{\circ}$ in ${ }^{\circ}$ intensity ${ }^{\circ}$ ratio $^{\circ}$ at the two $\mathrm{pH}$ values likely arises from charge repulsion. The D-Tyr substituted mutant adopts a more open conformation at either $\mathrm{pH}$ value compared to that of the wild-type Trp-cage at $\mathrm{pH} 7$. Our results offer further support ${ }^{\circ}$ of ${ }^{\circ}$ the ${ }^{\circ}$ proposal ${ }^{\circ}$ introduced ${ }^{\circ}$ by Adams ${ }^{\circ} t^{\circ} a l .{ }^{\circ}[13]$ that the substitution with the D-Tyr isomer within Trp-cage denatures the native Trp-cage conformation.

We further probed the Trp-cage by examining its conformation in an aqueous solution of 30\% TFE. Helixstabilizing effects of aqueous TFE are well documented [19]. ${ }^{\circ}$ Extensive ${ }^{\circ}$ work $^{\circ}$ on $^{\circ}$ helix-stabilizing ${ }^{\circ}$ effects ${ }^{\circ}$ of $^{\circ} \mathrm{TFE}$ indicate that $30 \% \mathrm{vol} / \mathrm{vol}$ is the optimum concentration of TFE needed 9 for helical'stabilization [1, 19]. Neidigh ${ }^{\circ}$ t al. ${ }^{\circ}[1]^{\circ}$ showed $^{\circ}$ that ${ }^{\circ} \operatorname{Trp}^{-c}$ age ${ }^{\circ}$ exhibits $^{\circ} a^{\circ}$ pronounced resistance to thermal denaturation in $30 \% \mathrm{vol} / \mathrm{vol}$ aqueous TFE solution. They reported an increased amide protection of Leu HN and Trp H $\epsilon 1$ hydrogens in TFE solution, which indicates a more stable native state. Based on this evidence Neidigh et al. argued that the addition of TFE led to increased "nativeness" of the protein.

Figure $2{ }^{\circ}$ shows ${ }^{\circ}$ ESI-ToF ${ }^{\circ}$ spectra ${ }^{\circ} f^{\circ} 40^{\circ} \mu \mathrm{M}^{\circ}$ Trp-cage in $30 \%$ TFE and $70 \% \mathrm{H}_{2} \mathrm{O}$ solution at $\mathrm{pH} 3$ and $\mathrm{pH}$ 7. The intensity ratio of +2 and +3 charge states at $\mathrm{pH} 7$ is 2.5 and the value is comparable to the value of 2.0 observed in aqueous solution. The stabilizing effect of TFE can be seen from the intensity ratio of +2 and +3 charge states at $\mathrm{pH} 3$. The ratio is 0.3 in pure water, whereas the ratio changes to 1.3 in $30 \%$ TFE aqueous solution. This signifies an increase in "nativeness" or compactness of Trp-cage in aqueous TFE solutions. Our conclusions agree $^{\circ}$ with $^{\circ}$ the ${ }^{\circ}$ observations ${ }^{\circ}$ of $^{\circ} \mathrm{Neidigh}^{\circ} \mathrm{et}^{\circ}$ al..$^{\circ}[1]$.

We further probed the Trp-cage conformation by examining the $\mathrm{H} / \mathrm{D}$ exchange protection of labile hydrogens. The spectrum of Trp-Cage in $100 \% \mathrm{H}_{2} \mathrm{O}$ is shown in Figure $3 a^{\circ}$ and ${ }^{\circ}{ }^{\circ} 100 \%{ }^{\circ} \mathrm{D}_{2} \mathrm{O}$, obtained within 1 $\min ^{\circ}$ after $^{\circ}$ dissolution, ${ }^{\circ}$ is ${ }^{\circ}$ shown $^{\circ}$ in $^{\circ}$ Figure $^{\circ} 3 b .^{\circ}$ The 

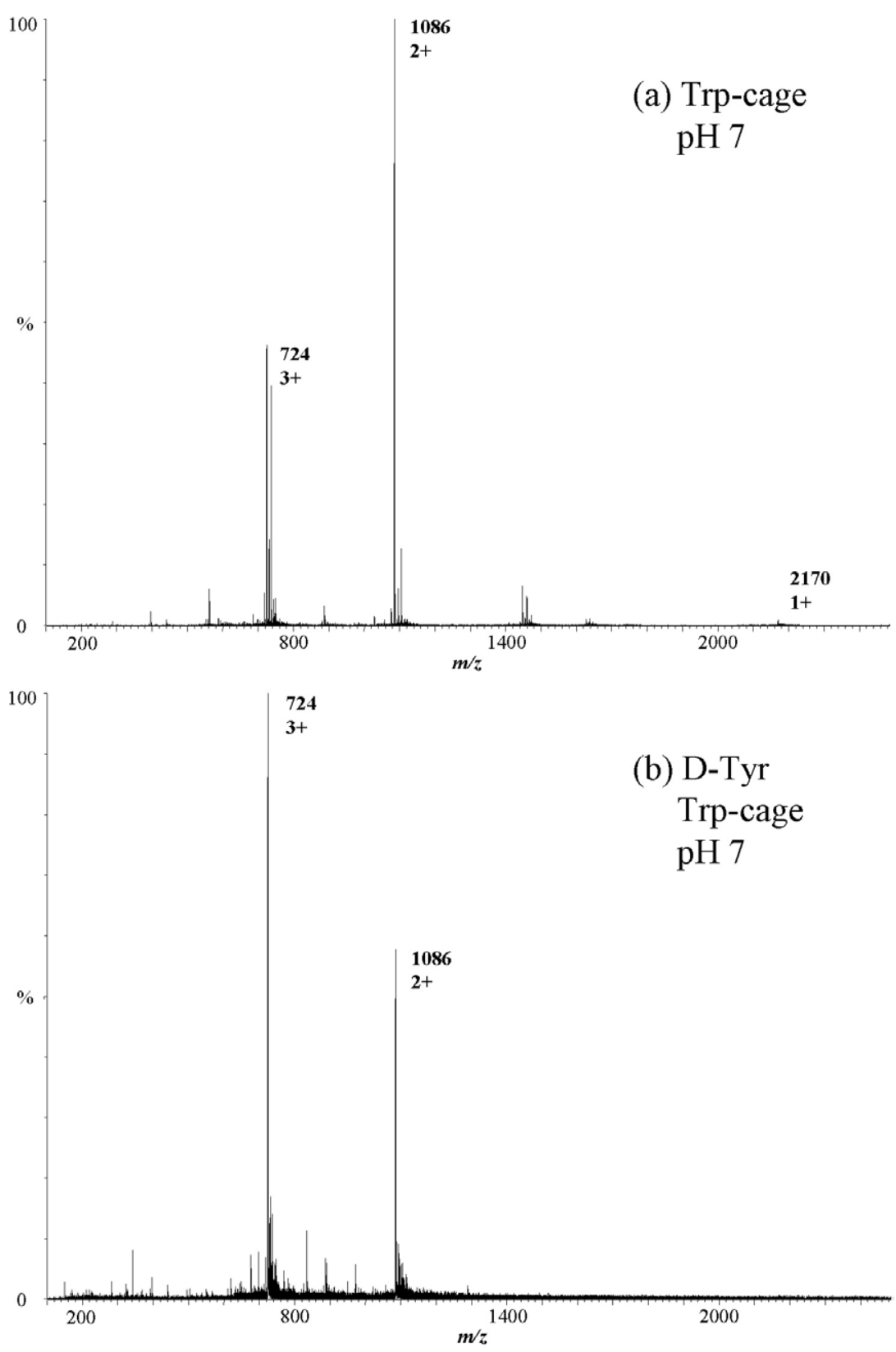

Figure 1. ESI-ToF spectrum of (a) $40 \mu \mathrm{M}$ Trp-cage in $\mathrm{H}_{2} \mathrm{O}$ at pH 7; (b) $18 \mu \mathrm{M}$ D-Tyr substituted Trp-cage in $\mathrm{H}_{2} \mathrm{O}$ at $\mathrm{pH} 7$.

Table 1. Intensity ratio of charge state $+2 /$ charge state +3 of Trp-cage and D-Tyr substituted Trp-cage under different solvent conditions

\begin{tabular}{lc}
\hline \multicolumn{1}{c}{ Experimental conditions } & Intensity of charge state +2 \\
\cline { 2 - 2 } & Intensity of charge state +3 \\
\hline \hline Trp-cage in $\mathrm{H}_{2} \mathrm{O}, \mathrm{pH} 3$ & 0.3 \\
Trp-cage in $\mathrm{H}_{2} \mathrm{O}, \mathrm{pH} 7$ & 2.0 \\
D-Tyr substituted Trp-cage in $\mathrm{H}_{2} \mathrm{O}, \mathrm{pH} 3$ & 0.2 \\
D-Tyr substituted Trp-cage in $\mathrm{H}_{2} \mathrm{O}, \mathrm{pH} \mathrm{7}$ & 0.6 \\
Trp-cage in 30\% TFE and 70\% $\mathrm{H}_{2} \mathrm{O}, \mathrm{pH} 3$ & 1.3 \\
Trp-cage in 30\% TFE and 70\% $\mathrm{H}_{2} \mathrm{O}, \mathrm{pH} \mathrm{7}$ & 2.5 \\
\hline
\end{tabular}



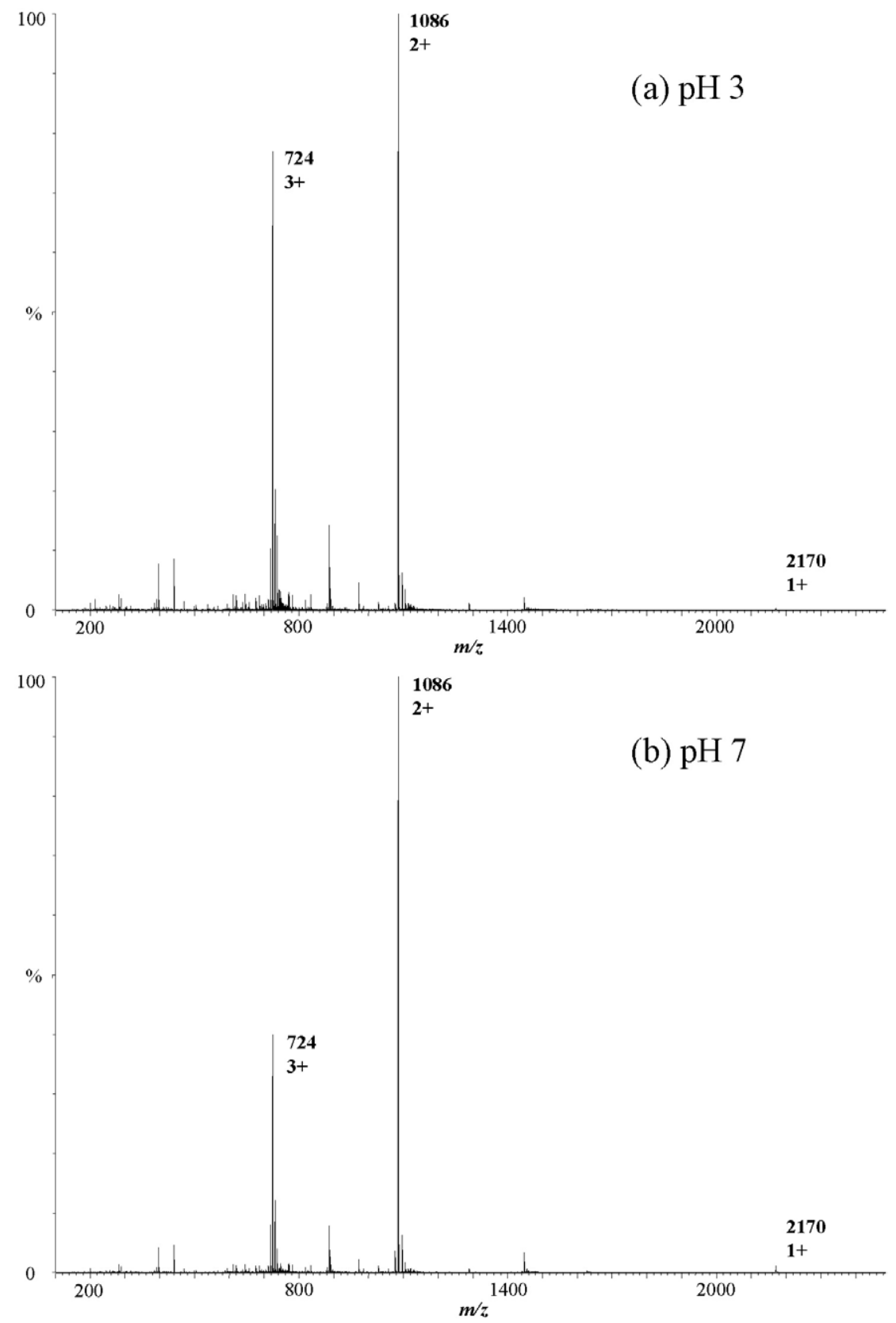

Figure 2. ESI-ToF spectrum of $40 \mu \mathrm{M}$ Trp-cage in $30 \%$ TFE and $70 \% \mathrm{H}_{2} \mathrm{O}$ solution at (a) $\mathrm{pH} 3$ and (b) $\mathrm{pH}$ 7. Some minor fragments arising from in-source fragmentation are observed.

number of labile hydrogens in Trp-cage is 34 , including two N-terminal hydrogens, 15 backbone amide hydrogens, one C-terminal hydrogen, and 16 side-chain hydrogens. The number of labile hydrogens on each side chain is indicated below:

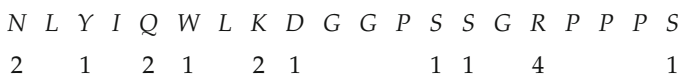

A total of 34 labile hydrogens are observed from comparing isotopic distribution of singly charged Trp-cage peak at $100 \% \mathrm{H}_{2} \mathrm{O}^{\circ}$ and $^{\circ} 100 \%{ }^{\circ} \mathrm{D}_{2} \mathrm{O}^{\circ}\left(\right.$ Figure $^{\circ} 3 \mathrm{a}^{\circ}$ and $^{\circ} \mathrm{b}$ ).
Note that a 35-mass-unit shift is observed as a result of the addition of a deuterium atom, instead of a proton, in the singly charged Trp-cage ion electrosprayed from $100 \% \mathrm{D}_{2} \mathrm{O}$. Likewise, an 18-mass-unit shift is observed in the doubly charged Trp-cage ion obtained from 100\% $\mathrm{D}_{2} \mathrm{O}$ as a result of the addition of two deuterium atoms.

The isotopic distribution of singly charged Trp-cage ions in $70 \% \mathrm{D}_{2} \mathrm{O}$ and $30 \%$ d-TFE is shown at 1- and 20-min ${ }^{\circ}$ intervals ${ }^{\circ}$ after $^{\circ}$ dissolution $^{\circ}$ in $^{\circ}$ Figure $^{\circ} 3 \mathrm{c}^{\circ}$ and ${ }^{\circ} \mathrm{d}$. After $20 \mathrm{~min}$, the isotopic distribution showed an increase of $\mathrm{m} / \mathrm{z}$ value by $1 \mathrm{amu}$ and is identical to the spectrum obtained from Trp-cage in $100 \% \mathrm{D}_{2} \mathrm{O}^{\circ}$ (Figure 

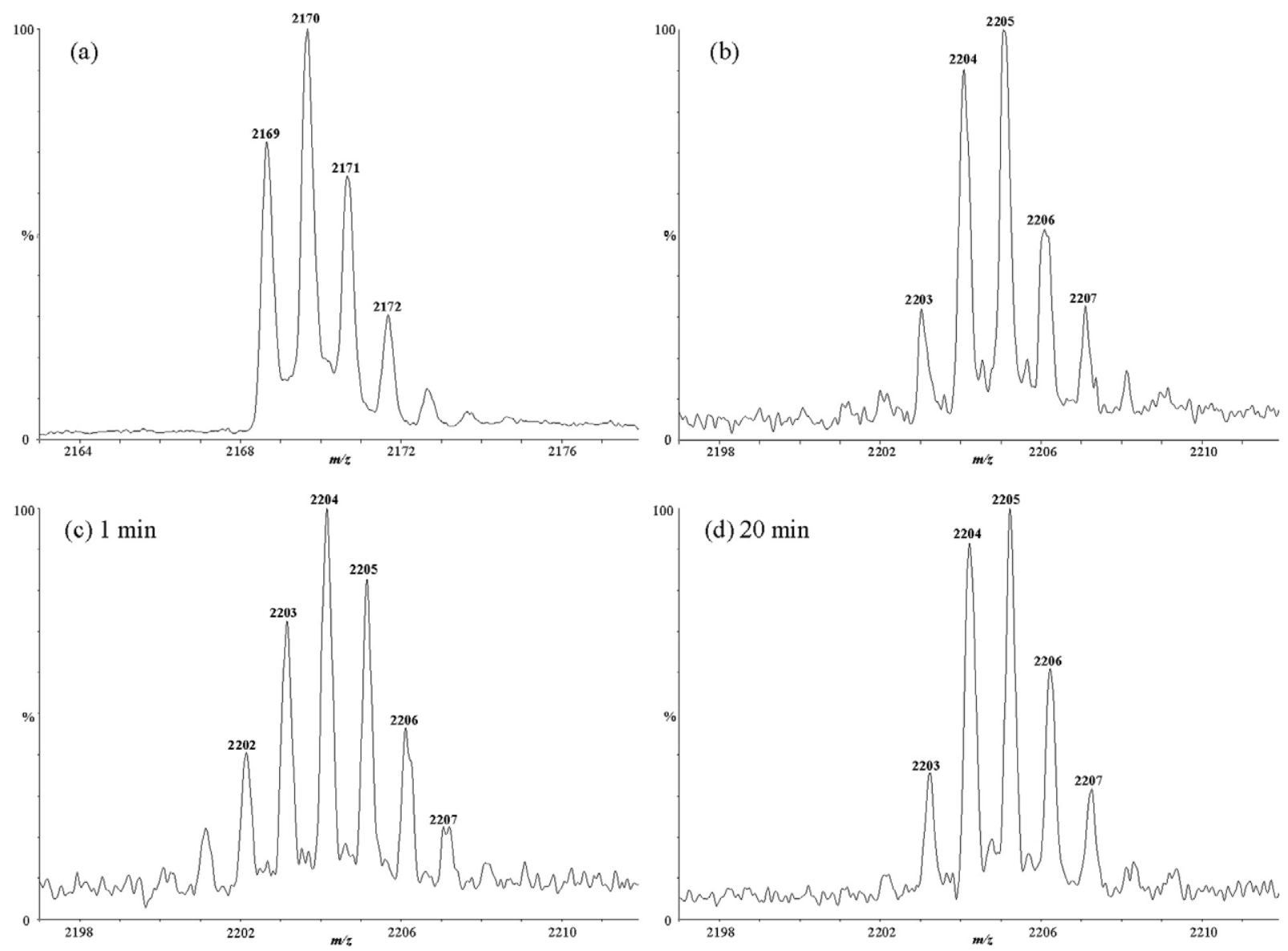

Figure 3. Isotopic distribution of singly charged Trp-cage in (a) $100 \% \mathrm{H}_{2} \mathrm{O}, \mathrm{pH}$; ; (b) $100 \% \mathrm{D}_{2} \mathrm{O}, \mathrm{pH}$ 7 ; (c) in $30 \%$ d-TFE $\mathrm{D}_{2} \mathrm{O}$ solution $\mathrm{pH} 7$ after 1-min incubation; (d) in $30 \%$ d-TFE $\mathrm{D}_{2} \mathrm{O}$ solution $\mathrm{pH} 7$ after 20-min incubation.

3b). ${ }^{\circ}$ Beyond ${ }^{\circ}$ the ${ }^{\circ} 20-\min ^{\circ} \operatorname{mark}^{\circ}$ the $^{\circ}$ spectrum $^{\circ}$ remains unchanged. The isotopic distribution of doubly charged Trp-cage ion obtained at 1 and 20 min yields the same result. Thus the time-dependent spectra of Trp-cage show increased H/D exchange protection of only one labile hydrogen atom. This increased protection against H/D exchange supports the TFE's stabilizing effect on Trp-cage's native state. The fact that native Trp-cage does not show any significant protection of the labile hydrogens in a water solution indicates that the native and nonnative unfolded conformations are separated by a small energy barrier. This allows for rapid interconversion between the folded and unfolded conformations. In $30 \%$ TFE solution, the rate of interconversion is sufficiently slowed down to afford some protection of the protein's labile hydrogen.

In this work we successfully applied ESI-MS CSD methodology to Trp-cage, which is one of the smallest known peptides to show conformationally dependent $\mathrm{CSD}^{\circ}$ shifts $^{\circ}\left[15-17,{ }^{\circ} 20\right]^{\circ}{ }^{\circ} \mathrm{Our}^{\circ}$ results $^{\circ}$ indicate ${ }^{\circ}$ that ${ }^{\circ} \mathrm{Trp}-$ cage conformation is $\mathrm{pH}$ sensitive and has maximum stability at around physiological $\mathrm{pH}$. The lowering of the $\mathrm{pH}$ beyond the $\mathrm{pH}$ 6-7 region disrupts ionic interaction in the native conformation leading to denatur- ation of Trp-cage. TFE solutions are shown to stabilize the native conformation and provide resistance against acid denaturation. This study demonstrates the utility of ESI-MS CSD in determining the compactness of tertiary proteins/polypeptides in a rapid and efficient manner.

\section{Acknowledgments}

The authors gratefully acknowledge NIH shared instrument grant 1S10RR017977-01 and NIH grant 8 RO1 EB002053201 for financial support, Professor Asher for providing the wild-type Trp-cage, and Professor Zubarev for providing the D-Tyr substituted Trpcage.

\section{References}

1. Neidigh, J. W.; Fesinmeyer, R. M.; Anderson, N. H. Designing a 20-residue protein. Nat. Struct. Biol. 2002, 9, 425-430.

2. Gellman, S. H.; Woolfson, D. N. Mini-proteins Trp the light fantastic. Nat. Struct. Biol. 2002, 9, 408-410.

3. Qiu, L.; Pabit, S. A.; Roitberg, A. E.; Hagen, S. J. Smaller and faster: The 20-residue Trp-cage protein folds in 14 microseconds. J. Am. Chem. Soc. 2002, 124, 12952-12953.

4. Simmerling, C.; Strockbine, B.; Roitberg, A. E. All-atom structure prediction and folding simulations of a stable protein. J. Am. Chem. Soc. 2002, 124, 11258-11259. 
5. Simmerling, C.; Strockbine, B.; Roitberg, A. E. Personal communication. Department of Chemistry, University of Florida, 2004.

6. Snow, C.; Zagrovic, B.; Pande, V. S. The Trp cage: Folding kinetics and unfolded state topology via molecular dynamics simulations. J. Am. Chem. Soc. 2002, 124, 14548-14549.

7. Chowdhury, S.; Lee, M. C.; Xiong, G.; Duan, Y. Ab initio folding simulation of the Trp-cage mini-protein approaches NMR resolution. I. Mol. Biol. 2003, 327, 711-717.

8. Zhou, R. Exploring the protein folding free energy landscape: Coupling replica exchange method with P3ME/RESPA algorithm. I. Mol. Graph. Model. 2004, 22, 451-462.

9. Zhou, R. Trp-cage: Folding free energy landscape in explicit water. Proc. Natl. Acad. Sci. USA 2003, 100, 13280-13285.

10. Chowdhury, S.; Lee, M. C.; Xiong, G.; Duan, Y. Characterizing the rate-limiting step of Trp-cage folding by all-atom molecular dynamics simulations. J. Phys. Chem. B 2004, 108, 13855-13865.

11. Pitera, J. W.; Swope, W. Understanding folding and design: Replicaexchange simulations of "Trp-cage" miniproteins. Proc. Natl. Acad. Sci. USA 2003, 100, 7587-7592

12. Ahmed, Z.; Beta, I. A.; Mikhonin, A. V.; Asher, S. A. UV-resonance Raman thermal unfolding study of Trp-cage shows that it is not a simple two-state miniprotein. I. Am. Chem. Soc. 2005, 127, 10943-10950.

13. Adams, C. M.; Kjeldsen, F.; Zubarev, R. A.; Budnik, B. A.; Haselmann, K. F. Electron capture dissociation distinguishes a single d-amino acid in a protein and probes the tertiary structure. J. Am. Soc. Mass Spectrom. 2004, 15, 1087-1098.

14. Grandori, R. Origin of the conformation dependence of protein chargestate distributions in electrospray ionization mass spectrometry. J. Mass Spectrom. 2003, 38, 11-15.

15. Grandori, R. Electrospray-ionization mass spectrometry for protein conformational studies. Curr. Org. Chem. 2003, 7, 1589-1603.

16. Samalikova, M.; Matecko, I.; Mueller, N.; Grandori, R. Interpreting conformational effects in protein nano-ESI-MS spectra. Anal. Bioanal. Chem. 2004, 378, 1112-1123.

17. Lin, H.; Dass, C. A mass spectrometry investigation of the conformational changes in adrenocorticotropic hormones. Eur. J. Mass Spectrom. 2002, 8, 381-387.

18. Kaltashov, I. A.; Eyles, S. J. Studies of biomolecular conformations and conformational dynamics by mass spectrometry. Mass Spectrom. Rev. 2002, 21, 37-71.

19. Lednev, I. K. K.; Anton, S.; Sparrow, M. C.; Asher, S. A. Alpha-helix peptide folding and unfolding activation barriers: A nanosecond UV resonance Raman study. J. Am. Chem. Soc. 1999, 121, 80748086.

20. Alomirah, H.; Alli, I.; Konishi, Y. Charge state distribution and hydrogen/deuterium exchange of $\alpha$-lactalbumin and $\beta$-lactoglobulin preparations by electrospray ionization mass spectrometry. J. Agric. Food Chem. 2003, 51, 2049-2057. 